\title{
Prescribed opioid analgesics in early pregnancy and the risk of congenital anomalies: a population-based cohort study
}

\author{
Alexa C. Bowie MPH, Martha M. Werler DSc, Maria P. Velez MD PhD, Wenbin Li MSc, Andi Camden MPH, \\ Astrid Guttmann MDCM MSc, Susan B. Brogly PhD MSc
}

Cite as: CMAJ 2022 February 7;194:E152-62. doi: 10.1503/cmaj.211215

\begin{abstract}
Background: Recent data suggest an increased risk of congenital anomalies with prenatal exposure to opioid analgesics. We sought to further quantify the risk of anomalies after opioid analgesic exposure during the first trimester in a population-based cohort study.
\end{abstract}

Methods: Using administrative health data from Ontario, we followed 599579 gestational parent-infant pairs from singleton pregnancies without opioid use disorder. We identified opioid analgesics dispensed in the first trimester and congenital anomalies diagnosed during the first year of life. We estimated propensity score-adjusted risk ratios (RRs) between first trimester exposure (any opioid analgesic and specific agents) and congenital anomalies (any anomaly, organ system anomalies, major or minor anomalies and specific anomalies).

Results: The prevalence of congenital anomalies was $2.8 \%$ in exposed infants and $2.0 \%$ in unexposed infants. Relative to unexposed infants, we observed elevated risks among those who were exposed for some anomaly groups, including gastrointestinal anomalies (any opioid analgesic: adjusted RR 1.46, 95\% confidence interval [Cl] 1.15-1.85; codeine: adjusted RR 1.53, 95\% Cl 1.12-2.09; tramadol: adjusted RR 2.69, 95\% $\mathrm{Cl} 1.34-5.38$ ) and several specific anomalies, including ankyloglossia (any opioid: adjusted RR 1.88, 95\% Cl 1.30-2.72; codeine: adjusted RR 2.14, 95\% Cl 1.35-3.40). These findings persisted in sensitivity analyses.

Interpretation: Although the absolute risk of congenital anomalies was low, our findings add to accumulating data that suggest a small increased risk of some organ system anomalies and specific anomalies with first trimester exposure to opioid analgesics. These findings further quantify the potential risks associated with prenatal exposure to opioid analgesics to inform treatment choices for pain in pregnancy.

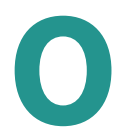

pioid use in pregnancy is a public health concern, ${ }^{1-5}$ with $2 \%-4 \%$ of pregnancies exposed to prescribed opioid analgesics..$^{6-8}$ Opioid analgesics cross the placenta and have potential to cause fetal harm. ${ }^{9}$

Evidence concerning the safety of opioid analgesics for pain in pregnancy is discrepant and limited. ${ }^{10-13}$ Recent studies with Medicaid and private insurance health data from the United States suggested small increases in minor congenital anomalies and oral cleft anomalies with prenatal exposure to opioid analgesics..$^{10,14}$ Some studies have reported an increased risk of any anomaly, ${ }^{15,16}$ heart anomalies, ${ }^{7,16-18}$ spina bifida, ${ }^{7,19,20}$ oral cleft anomalies, ${ }^{10,15,21}$ gastroschisis ${ }^{7}$ and clubfoot ${ }^{16,22,23}$ with exposure. Other studies, however, have found no association with any anomaly, ${ }^{22,24}$ major $^{10,14,19,22,24,25}$ or minor ${ }^{14}$ anomalies, neural tube anomalies, ${ }^{10,19,22,26}$ clubfoot $^{10}$ or heart anomalies. ${ }^{8,15,19,22,27,28}$ Discrepant findings likely arise from different methodologies, including definitions of exposure and anomalies evaluated. Some studies included pregnant people with exposure to opioid agonist therapy and, therefore, opioid use disorder. ${ }^{20,22,23}$ Many studies captured exposure through maternal self-report. $7,8,15-18,20-24,26,28,29$ Others predominantly included births before $1990,8,15,17,18,21,25,27,28$ preventing evaluation of current medications. Only 2 population-based cohort studies have been conducted. ${ }^{22,24}$

Therefore, we undertook a population-based cohort study to estimate associations between opioid analgesic exposure during the first trimester and congenital anomalies using health administrative data capturing all narcotic prescriptions during pregnancy.

\section{Methods}

\section{Study design and data sources}

We constructed a population-based cohort of deliveries using administrative health data of the single-payer health care system in Ontario, Canada, held at ICES. We identified parent-infant pair 
records for all live births and stillbirths at more than 20 weeks' gestation delivered at Ontario hospitals with an estimated date of confinement between Apr. 7, 2013 (280 d from implementation of the Narcotic Monitoring System [NMS]), and Mar. 31, 2018, in MOMBABY, a validated database of linked parent-infant records. We used estimated date of confinement rather than delivery date to prevent overselection of preterm births and, thus, anomalies. ${ }^{30}$ To reduce confounding, we excluded pregnant people with opioid use disorder or opioid overdose within 2 years before delivery or those treated with methadone or buprenorphine for opioid use disorder. ${ }^{1,31,32}$ We also excluded pairs without a valid Ontario health card, pairs with unsuccessful record linkage, pregnant people aged 50 and older, those with multiple fetuses and pregnancies exposed to opioid analgesics during the second or third trimester, but not the first.

\section{Exposure}

We captured exposure using records of prescribed prenatal opioid analgesics in the NMS database. Established in July 2012 through the Ontario Narcotics Strategy, the NMS records information on controlled drug prescriptions issued to Ontario residents, regardless of payment method. The exposure of interest was to opioid analgesics during the first trimester, defined as a prescription fill date between the estimated date of conception (following a validated algorithm;33 Appendix 1, available at www.cmaj.ca/lookup/ doi/10.1503/cmaj.211215/tab-related-content) and less than 14 weeks' gestation. We classified exposure as any opioid analgesic and specific agents. The referent group was unexposed to any opioid analgesic during the index pregnancy period.

\section{Outcomes}

We identified congenital anomalies diagnosed within the first year of life using diagnosis codes from the Canadian version of the International Classification of Diseases, 10th Revision in the Hospital Discharge Abstract Database, the Same Day Surgery Database and the National Ambulatory Care Reporting System Database, which record mandatory hospital submissions (Appendix 1, Supplemental eTable 1). As in other studies, ${ }^{34-36}$ we classified anomalies using the algorithm from the Metropolitan Atlanta Congenital Defects Program, a surveillance system of the US Centers for Disease Control and Prevention (Appendix 1, Supplemental eTable 2). ${ }^{37,38}$ We defined anomalies as major (of medical or surgical importance) or minor (associated with minor medical or cosmetic significance). We classified them as any anomaly, by organ system, major or minor ${ }^{37}$ and specific anomalies if at least 5 infants with the anomaly were exposed.

\section{Confounders}

We computed high-dimensional propensity scores (HDPS) for parent-infant pairs to ensure similarity of pairs by exposure. This method was developed for pharmacoepidemiologic administrative health data studies ${ }^{39}$ and has been used with ICES data. ${ }^{6,40,41}$ Briefly, using the HDPS algorithm, candidate covariates in the year before conception were identified empirically in the health care claims databases we selected (Appendix 1, Supplemental eTable 1). We prioritized covariates by their potential to control for confounding, and integrated them into an exposure propensity score. ${ }^{39}$ We forced the following a priori confounders into the HPDS: gestational parent age, parity, socioeconomic status quintile, Elixhauser comorbidity score, diabetes, obesity, hypertension, pain, other prescribed psychotropic medications (only data on benzodiazepines or barbiturates were available in the NMS), and year of delivery. The resultant HDPS (between 0 and 1 ) for each parent-infant pair represents their probability of being treated with opioid analgesics conditional on the confounders. We further stabilized the HDPS to account for extreme weights. ${ }^{42}$

\section{Statistical analysis}

Given the large study size, we compared gestational parentinfant characteristics by exposure with standardized differences; we considered differences greater than 0.10 statistically meaningful. ${ }^{43}$ We estimated risk ratios (RR) between opioid analgesic exposure during the first trimester and any congenital anomaly, organ system anomalies, major or minor anomalies, and specific anomalies using a log-binomial regression model. We estimated adjusted risk ratios using inverse probability of treatment weighting with HDPS.

\section{Sensitivity analysis}

Sensitivity analyses included redefining the exposure period to include the 4 weeks before conception to evaluate possible misclassification; excluding individuals with opioid analgesic use in the year before pregnancy for possible confounding; analyzing individuals with more than 1 pregnancy, for which at least 1 was exposed and 1 was unexposed, to evaluate confounding; excluding infants born earlier than 37 weeks' gestation (for analysis of atrial septal defect, hypertrophic pyloric stenosis, ankyloglossia possibly related to prematurity); and stratifying by sex for effect measure modification (for hypertrophic pyloric stenosis). Additionally, we estimated adjusted risk differences to provide information on absolute effect size. Finally, we used 2 methods to explore the likely effect of potential unmeasured confounding. First, we determined the effect of an unmeasured confounder needed to fully account for our observed increased risk. ${ }^{44}$ Second, we used simple bias analysis ${ }^{45}$ to further remove possible unmeasured confounding arising from incomplete data on prenatal medications; we used estimates from previous studies (confounder-outcome association 1.5-3.0, and prevalence of psychotropic medication use by exposure group). ${ }^{46}$

\section{Ethics approval}

This study was approved by the Queen's University Health Sciences Research Ethics Board.

\section{Results}

Of 651180 births in Ontario during the study period, 599579 (92.1\%) were included in the study cohort (Figure 1). The 599579 pregnancies occurred among 491060 individuals, 111055 (22.6\%) of whom were nulliparous. Of the 599579 pregnancies, 11903 (2.0\%) were exposed to opioid analgesics. Most 


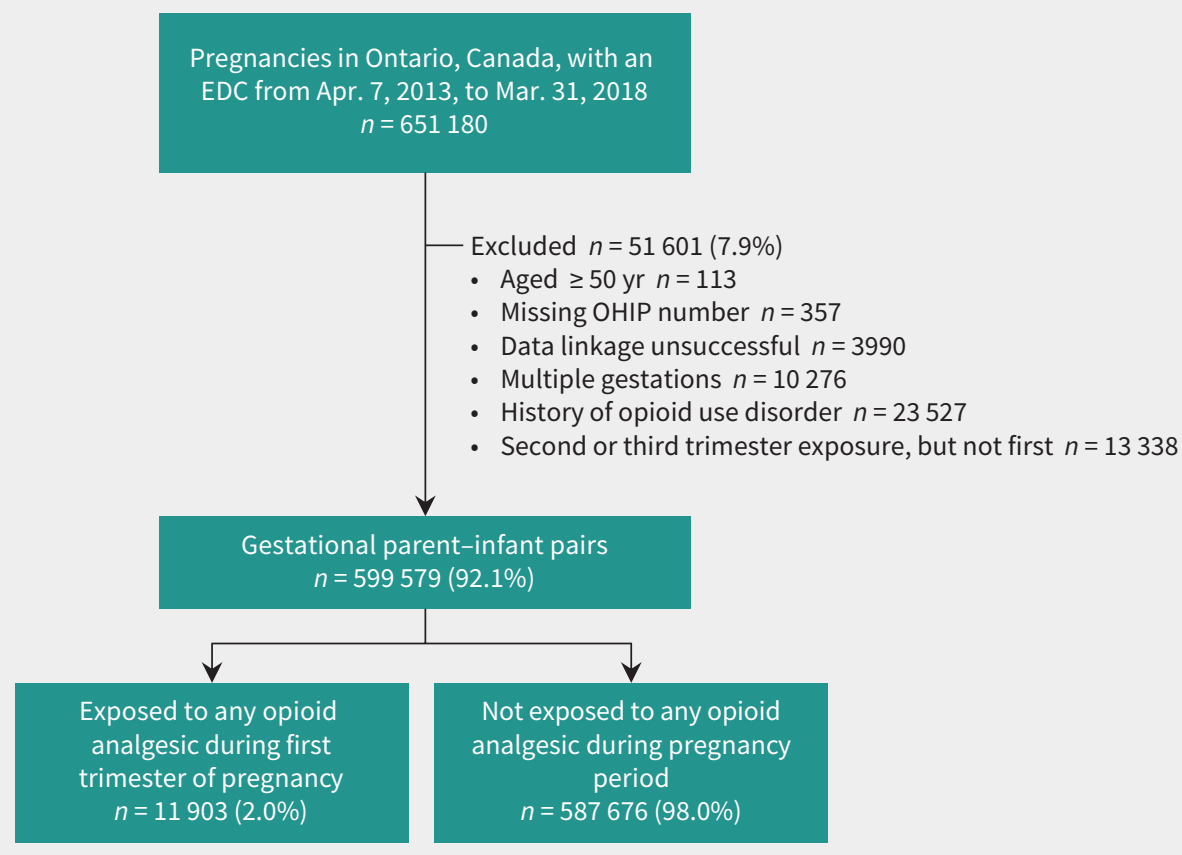

Figure 1: Flow chart of cohort creation. Note: $E D C=$ estimated date of conception, $\mathrm{OHIP}=$ Ontario Health Insurance Plan.

pairs were exposed during the first trimester only $(n=9023$, $75.8 \%)$ with 1195 (10.0\%) exposed all 3 trimesters; the mean duration of exposure was 4.3 (standard deviation 9.2) weeks. Specific agents included codeine $(n=6524)$, oxycodone ( $n=2885)$, hydromorphone $(n=1824)$, tramadol $(n=781)$, morphine $(n=670)$, fentanyl $(n=75)$, meperidine $(n=56)$ and others $(n=41)$. Standardized differences (Table 1 ) showed that exposed pairs were more likely to have been prescribed opioid analgesics in the year before pregnancy ( $47.6 \%$ v. $10.1 \%)$, to have a prescription for other psychotropics (8.4\% v. $1.4 \%)$, and to have an Elixhauser comorbidity score of 1 or more (2.2\% v. $0.7 \%)$ than those who were unexposed. Those exposed were also more likely to have a prior maternal diagnosis of pain ( $26.3 \%$ v. $9.8 \%)$, particularly lower back pain (20.9\% v. $7.9 \%)$. Most covariates were balanced after HDPS weighting, except for history of opioid analgesic use and prescribed psychotropics.

Overall, 12260 (2.0\%) infants received a congenital anomaly diagnosis in the first year of life; 329 (2.8\%) of 11903 exposed infants received a diagnosis of an anomaly, compared with 11931 $(2.0 \%)$ of 587676 unexposed infants. The number of anomalies per infant was similar by exposure group; 230 (69.9\%) exposed infants with anomalies had 1 anomaly, compared with 7973 (66.8\%) of those unexposed (Appendix 1, Supplemental eTable 3).

Figure 2 shows the unadjusted and adjusted RRs between exposure to opioid analgesics in the first trimester and any anomaly. After HDPS adjustment, infants exposed to any opioid analgesic had a small, elevated risk of any congenital anomaly (adjusted RR 1.14, 95\% confidence interval [Cl] 1.01-1.28) for an adjusted prevalence difference of 2.9 per 1000 infants (Appendix 1,
Supplemental eTable 4). We observed an increased risk of any anomaly with exposure to morphine (adjusted RR $1.89,95 \% \mathrm{Cl}$ 1.28-2.79) and tramadol (adjusted RR 1.79, 95\% Cl 1.23-2.60).

When classified by organ system and specific anomalies (Figure 3, Figure 4 and Figure 5), exposure was associated with increased risks of cardiovascular anomalies (with morphine), neoplasms and tumours (with tramadol), gastrointestinal (with any opioid analgesic and with codeine) and genital anomalies (with oxycodone). The risk of urinary anomalies was lower with any opioid analgesic exposure (adjusted RR 0.63 , 95\% $\mathrm{Cl} 0.41-0.96$ ) and higher with tramadol (adjusted RR 2.95, 95\% Cl 1.22-7.14).

The risk of major anomalies was elevated with exposure to morphine (adjusted RR 2.05, 95\% 1.34-3.13, Figure 4) and tramadol (adjusted RR 1.94, 95\% Cl 1.28-2.92). Associations with specific major anomalies included atrial septal defect (with tramadol), ventricular septal defect (with codeine), pulmonary artery stenosis (with any opioid analgesic and with codeine) and hypertrophic pyloric stenosis (with any opioid analgesic, codeine and morphine). The risk of minor anomalies was elevated with exposure to any opioid analgesic, codeine, hydromorphone and oxycodone, and the risk of ankyloglossia was elevated with any opioid analgesic and codeine (Figure 5).

Specific agents associated with multiple anomalies included tramadol (with atrial septal defect, neoplasms and tumours, gastrointestinal anomalies and urinary anomalies), codeine (with gastrointestinal anomalies, ankyloglossia, hypertrophic pyloric stenosis, ventricular septal defect and pulmonary artery stenosis) and morphine (with cardiovascular anomalies and hypertrophic pyloric stenosis). Only oxycodone was associated with a single anomaly group (genital anomalies). 
Table 1: Characteristics of pregnancies exposed to opioid analgesics in the first trimester, compared with pregnancies unexposed during the pregnancy period in Ontario, Canada, 2013-2018

\begin{tabular}{|c|c|c|c|c|c|c|}
\hline \multirow[b]{2}{*}{ Characteristic } & \multicolumn{3}{|c|}{ Before weighting } & \multicolumn{3}{|c|}{ After weighting } \\
\hline & $\begin{array}{c}\text { No. }(\%) \\
\text { exposed } \\
n=11903\end{array}$ & $\begin{array}{c}\text { No. }(\%) \\
\text { unexposed } \\
n=587676\end{array}$ & $\begin{array}{l}\text { Standardized } \\
\text { difference }\end{array}$ & $\begin{array}{l}\text { No. }(\%) \\
\text { exposed }\end{array}$ & $\begin{array}{l}\text { No. }(\%) \\
\text { unexposed }\end{array}$ & $\begin{array}{l}\text { Standardized } \\
\text { difference* }\end{array}$ \\
\hline $\begin{array}{l}\text { Opioid analgesic use in the year } \\
\text { before pregnancy }\end{array}$ & $5662(47.6)$ & $59108(10.1)$ & 0.91 & $3757(31.6)$ & $61941(10.5)$ & 0.533 \\
\hline \multicolumn{7}{|l|}{ Maternal age at delivery, yr } \\
\hline$<20$ & $245(2.1)$ & $12049(2.1)$ & 0.001 & $245(2.1)$ & $12047(2.1)$ & 0.000 \\
\hline $20-24$ & $1502(12.6)$ & $60568(10.3)$ & 0.073 & $1459(12.3)$ & $61001(10.4)$ & 0.059 \\
\hline $25-29$ & $3135(26.3)$ & 158052 (26.9) & 0.013 & $3203(26.9)$ & 157967 (26.9) & 0.001 \\
\hline $30-34$ & $4021(33.8)$ & $218741(37.2)$ & 0.072 & $4132(34.7)$ & 218263 (37.1) & 0.051 \\
\hline$\geq 35$ & $3000(25.2)$ & $138266(23.5)$ & 0.039 & $2864(24.1)$ & 138339 (23.5) & 0.012 \\
\hline \multicolumn{7}{|l|}{ Year of delivery } \\
\hline 2013 & $2023(17.0)$ & $91418(15.6)$ & 0.039 & $1874(15.7)$ & $91384(15.6)$ & 0.005 \\
\hline 2014 & $2510(21.1)$ & $117456(20.0)$ & 0.027 & $2219(18.6)$ & $117947(20.1)$ & 0.036 \\
\hline 2015 & $2505(21.0)$ & $117114(19.9)$ & 0.028 & $2426(20.4)$ & $117241(20.0)$ & 0.010 \\
\hline 2016 & $2398(20.2)$ & $118223(20.1)$ & 0.001 & $2514(21.1)$ & $117947(20.1)$ & 0.026 \\
\hline 2017 & $2087(17.5)$ & $118402(20.1)$ & 0.067 & $2371(19.9)$ & 118231 (20.1) & 0.004 \\
\hline 2018 & $380(3.2)$ & $25063(4.3)$ & 0.057 & $500(4.2)$ & $24976(4.3)$ & 0.003 \\
\hline \multicolumn{7}{|l|}{ SES quintile at delivery } \\
\hline 1-2 (lowest) & $5863(49.3)$ & $250350(42.6)$ & 0.133 & $5118(43.0)$ & $251702(42.8)$ & 0.003 \\
\hline 3 & $2394(20.1)$ & $120650(20.5)$ & 0.010 & $2406(20.2)$ & $120356(20.5)$ & 0.007 \\
\hline 4 & 2131 (17.9) & 120826 (20.6) & 0.067 & $2422(20.4)$ & $120297(20.5)$ & 0.003 \\
\hline 5 (highest) & $1515(12.7)$ & 95909 (16.3) & 0.102 & $1957(16.4)$ & $95321(16.2)$ & 0.006 \\
\hline \multicolumn{7}{|l|}{ Maternal conditions ${ }^{\dagger}$} \\
\hline Diabetes & $453(3.8)$ & $9446(1.6)$ & 0.136 & 312 (1.9) & $14457(1.7)$ & 0.019 \\
\hline Obesity & $571(4.8)$ & $14093(2.4)$ & 0.129 & $268(2.62)$ & $12811(2.5)$ & 0.010 \\
\hline Hypertension & $482(4.0)$ & $12547(2.1)$ & 0.110 & $1383(2.25)$ & $60354(2.2)$ & 0.005 \\
\hline \multicolumn{7}{|l|}{ Maternal pain diagnosis $\dagger$} \\
\hline Any & $3131(26.3)$ & 57393 (9.8) & 0.440 & 1383 (11.6) & $603540(10.3)$ & 0.034 \\
\hline Lower back & 2491 (20.9) & 46146 (7.9) & 0.379 & $1096(9.2)$ & 48601 (8.3) & 0.036 \\
\hline Migraine & $451(3.8)$ & $5256(0.9)$ & 0.192 & $160(1.3)$ & $5642(1.0)$ & 0.019 \\
\hline Chronic & 364 (3.1) & $5163(0.9)$ & 0.157 & $135(1.1)$ & $5524(0.9)$ & 0.017 \\
\hline Other & $239(2.0)$ & $1897(0.3)$ & 0.158 & $58(0.5)$ & $2233(0.4)$ & 0.004 \\
\hline Limb & 196 (1.6) & $2880(0.5)$ & 0.113 & $74(0.6)$ & $3467(0.6)$ & 0.006 \\
\hline Arthritis & $76(0.6)$ & $1617(0.3)$ & 0.054 & $30(0.3)$ & $1645(0.3)$ & 0.002 \\
\hline Facial & $47(0.4)$ & $891(0.2)$ & 0.047 & $19(0.2)$ & $940(0.2)$ & 0.003 \\
\hline Elixhauser comorbidity score $\geq 1 \dagger$ & $266(2.2)$ & $4083(0.7)$ & 0.13 & $105(0.9)$ & $4349(0.7)$ & 0.016 \\
\hline Previous live birth & $2001(16.8)$ & 109054 (18.6) & 0.05 & $2237(18.8)$ & $108720(18.5)$ & 0.007 \\
\hline $\begin{array}{l}\text { Other prescribed psychotropic } \\
\text { medications } \ddagger\end{array}$ & $998(8.4)$ & $7934(1.4)$ & 0.33 & $480(4.0)$ & $8580(1.5)$ & 0.158 \\
\hline
\end{tabular}




\begin{tabular}{llccccr} 
Anomaly & \multicolumn{1}{c}{ Exposure* } & $\begin{array}{c}\text { No. of } \\
\text { infants }\end{array}$ & $\begin{array}{c}\text { No. of infants } \\
\text { with anomaly }\end{array}$ & Unadjusted & Adjusted \\
\hline Any congenital & None & 587676 & 11931 & 1.00 & 1.00 \\
anomaly & Any & 11903 & 329 & $1.37(1.23-1.53)$ & $1.14(1.01-1.28)$ \\
& Codeine & 6524 & 160 & $1.21(1.04-1.42)$ & $1.10(0.94-1.30)$ \\
& Morphine & 670 & 25 & $1.87(1.25-2.79)$ & $1.89(1.28-2.79)$ \\
& Hydromorphone & 1824 & 49 & $1.33(1.00-1.77)$ & $1.12(0.82-1.53)$ \\
& Oxycodone & 2885 & 87 & $1.50(1.21-1.86)$ & $1.14(0.89-1.46)$ \\
& Tramadol & 781 & 38 & $2.47(1.78-3.42)$ & $1.79(1.23-2.60)$
\end{tabular}

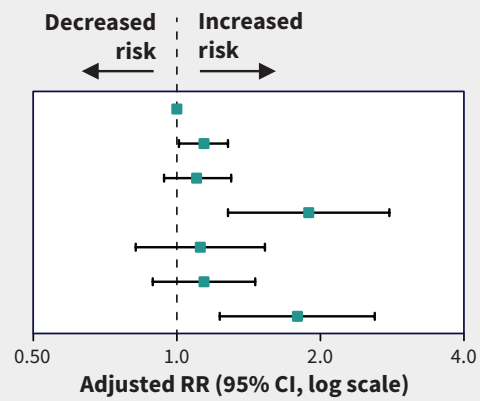

Figure 2: Forest plot of the risk of any congenital anomaly in pregnancies exposed to opioid analgesics in the first trimester compared with those not exposed during the pregnancy period, by specific agent. We adjusted estimates using inverse probability of treatment weighting with high-dimensional propensity scores. *We present data on specific opioid analgesic agents where at least 5 infants with the anomaly were exposed. Note: $\mathrm{Cl}=$ confidence interval, $\mathrm{RR}=$ risk ratio.

In sensitivity analyses (Table 2), when including exposure 4 weeks before conception or excluding individuals with exposure to opioid analgesics before pregnancy, results were unchanged from the primary analyses. Among 2980 individuals with at least 1 exposed and 1 unexposed pregnancy, exposure to any opioid analgesic was associated with an increased risk of any anomaly (adjusted RR 1.13, 95\% Cl 0.83-1.55). An increased risk of ankyloglossia and hypertrophic pyloric stenosis with exposure to any opioid analgesic remained when restricting to term infants. We observed a stronger association between any opioid analgesic exposure and hypertrophic pyloric stenosis in female infants (adjusted RR 3.97, 95\% Cl 2.11-7.50) than male infants (adjusted RR $1.49,95 \% \mathrm{Cl} 0.92-2.41)$. For confounding to fully account for our observed increased risk, most observed associations required risk ratios greater than 3 between the possible unmeasured confounder and the exposure and anomalies (Appendix 1, Supplemental eTable 5). Most findings persisted after further adjustment for possible unmeasured confounding (Appendix 1, Supplemental eTable 6).

\section{Interpretation}

In this population-based cohort of 599579 parent-infant pairs, the prevalence of congenital anomalies was 204.4 per 10000 infants; this is lower than the prevalence of 430.5 per 10000 infants in the 2014 general Canadian pregnancy population. ${ }^{47}$ Although the overall risk was low, we observed an increased risk of any congenital anomaly with tramadol, ${ }^{16}$ and a previously unreported risk with morphine. An association with oxycodone was observed only when including exposure 4 weeks before conception. Associations with any anomaly, however, are less informative: any anomaly is a crude classification and may not capture differences in etiology. ${ }^{48}$

Previous studies reported elevated risks of heart anomalies with first trimester exposure to any opioid analgesic, ${ }^{7}$ codeine $^{7,17,18}$ and tramadol, ${ }^{16}$ but others reported no association with any opioid analgesic ${ }^{8,10,15,19,22}$ or codeine. ${ }^{10,19,22,27,28}$ A US case-control study reported increased risks of specific heart anomalies with periconceptional or first trimester exposure to opioid analgesics compared with acetaminophen. ${ }^{29}$ Like others, ${ }^{7,16-18}$ we observed associations with some cardiovascular anomalies; however, these did not persist in bias analysis.

Few studies have evaluated hypertrophic pyloric stenosis, $15,16,22$ a major stomach anomaly leading to gastric outlet obstruction that requires surgery ${ }^{49}$ we observed an increased risk with any opioid analgesic, codeine and morphine. We observed a stronger association among female infants than male infants, which persisted in bias analysis. Hypertrophic pyloric stenosis is more common in male than female infants..$^{50,51}$ It is unknown why the baseline risk differs by sex; similarly, we cannot explain why sex would modify associations with exposure to opioid analgesics. This observation may be hypothesisgenerating rather than suggestive of opioid analgesic teratogenicity.

Others have observed associations with exposure to codeine in the second and third trimesters and to oxycodone in the third trimester. ${ }^{14}$ We observed an elevated risk of minor anomalies with any opioid analgesic, codeine, oxycodone and hydromorphone; associations were strongest for hydromorphone in bias analysis. We noted associations between any opioid analgesic and codeine with ankyloglossia, commonly known as tongue-tie, which is sometimes treated with release of the frenulum. We also observed other elevated risks: gastrointestinal anomalies with any opioid analgesic, codeine and tramadol; genital anomalies with oxycodone; and neoplasms and tumours and urinary anomalies with tramadol. We attribute the observed lower risk of urinary anomalies with any opioid analgesic to type I error. The difficulty of accurate exposure measurement is that etiology and time of organogenesis can vary within an organ system, which could reduce the specificity of estimated associations. ${ }^{48}$

Multiple sensitivity analyses suggested that misclassification of exposure or anomalies likely did not account for our results. Our HDPS adjustment attenuated some estimates, but elevated associations remained for some anomalies. Consistent with others, ${ }^{10}$ our quantitative bias analysis estimated that an unmeasured confounder would have to be strongly associated with exposure and anomalies to fully account for associations. To correct estimates for possible unmeasured confounding from psychotropic medications (only benzodiazepines and barbiturates are available in the NMS and 


\begin{tabular}{|c|c|c|c|c|c|}
\hline \multirow[b]{2}{*}{ Organ system } & \multirow[b]{2}{*}{ Exposure* } & \multirow{2}{*}{$\begin{array}{l}\text { No. of } \\
\text { infants }\end{array}$} & \multirow{2}{*}{$\begin{array}{l}\text { No. of infants } \\
\text { with anomaly }\end{array}$} & \multicolumn{2}{|c|}{ RR $(95 \% \mathrm{Cl})$} \\
\hline & & & & Unadjusted & Adjusted \\
\hline \multirow[t]{7}{*}{ Cardiovascular } & None & 587676 & 3130 & 1.00 & 1.00 \\
\hline & Any & 11903 & 83 & $1.31(1.05-1.63)$ & $1.13(0.89-1.43)$ \\
\hline & Codeine & 6524 & 45 & $1.30(0.97-1.74)$ & $1.19(0.88-1.63)$ \\
\hline & Morphine & 670 & 7 & $1.97(0.94-4.16)$ & $2.23(1.10-4.52)$ \\
\hline & Hydromorphone & 1824 & 8 & $0.82(0.41-1.65)$ & $0.79(0.39-1.63)$ \\
\hline & Oxycodone & 2885 & 20 & $1.30(0.84-2.03)$ & $0.64(0.34-1.24)$ \\
\hline & Tramadol & 781 & 11 & $2.68(1.47-4.84)$ & $1.45(0.63-3.31)$ \\
\hline \multirow[t]{7}{*}{ Gastrointestinal } & None & 587676 & 2395 & 1.00 & 1.00 \\
\hline & Any & 11903 & 79 & $1.63(1.30-2.04)$ & $1.46(1.15-1.85)$ \\
\hline & Codeine & 6524 & 41 & $1.55(1.13-2.11)$ & $1.53(1.12-2.09)$ \\
\hline & Morphine & 670 & 8 & $2.95(1.47-5.94)$ & $2.05(0.88-4.77)$ \\
\hline & Hydromorphone & 1824 & 7 & $0.94(0.45-1.98)$ & $1.09(0.54-2.20)$ \\
\hline & Oxycodone & 2885 & 20 & $1.71(1.10-2.65)$ & $1.15(0.65-2.00)$ \\
\hline & Tramadol & 781 & 10 & $3.17(1.70-5.92)$ & $2.69(1.34-5.38)$ \\
\hline \multirow[t]{5}{*}{ Musculoskeletal } & None & 587676 & 1997 & 1.00 & 1.00 \\
\hline & Any & 11903 & 54 & $1.34(1.02-1.75)$ & $1.02(0.76-1.37)$ \\
\hline & Codeine & 6524 & 26 & $1.17(0.80-1.73)$ & $1.25(0.85-1.82)$ \\
\hline & Hydromorphone & 1824 & 9 & $1.45(0.75-2.80)$ & $1.49(0.77-2.88)$ \\
\hline & Oxycodone & 2885 & 16 & $1.64(1.00-2.68)$ & $1.53(0.90-2.60)$ \\
\hline \multirow[t]{4}{*}{ Genital } & None & 587676 & 1247 & 1.00 & 1.00 \\
\hline & Any & 11903 & 28 & $1.11(0.76-1.61)$ & $1.07(0.73-1.58)$ \\
\hline & Codeine & 6524 & 12 & $0.87(0.49-1.53)$ & $0.79(0.43-1.45)$ \\
\hline & Oxycodone & 2885 & 11 & $1.80(0.99-3.26)$ & $2.36(1.37-4.06)$ \\
\hline \multirow[t]{6}{*}{ Urinary } & None & 587676 & 1351 & 1.00 & 1.00 \\
\hline & Any & 11903 & 32 & $1.17(0.82-1.66)$ & $0.63(0.41-0.96)$ \\
\hline & Codeine & 6524 & 12 & $0.80(0.45-1.41)$ & $0.74(0.41-1.35)$ \\
\hline & Hydromorphone & 1824 & 7 & $1.67(0.79-3.52)$ & $0.66(0.20-2.19)$ \\
\hline & Oxycodone & 2885 & 10 & $1.51(0.81-2.82)$ & $1.10(0.52-2.35)$ \\
\hline & Tramadol & 781 & $5-6$ & $2.80(1.16-6.75)$ & $2.95(1.22-7.14)$ \\
\hline \multirow[t]{4}{*}{ Neoplasms and tumours } & None & 587676 & 886 & 1.00 & 1.00 \\
\hline & Any & 11903 & 22 & $1.23(0.80-1.87)$ & $1.14(0.73-1.78)$ \\
\hline & Codeine & 6524 & 11 & $1.12(0.62-2.03)$ & $1.07(0.58-1.98)$ \\
\hline & Tramadol & 781 & $5-6$ & $4.27(1.77-10.31)$ & $4.71(1.98-11.17)$ \\
\hline \multirow[t]{4}{*}{ Central nervous system } & None & 587676 & 735 & 1.00 & 1.00 \\
\hline & Any & 11903 & 21 & $1.41(0.91-2.18)$ & $1.01(0.61-1.68)$ \\
\hline & Codeine & 6524 & 7 & $0.86(0.41-1.81)$ & $0.67(0.29-1.57)$ \\
\hline & Oxycodone & 2885 & 8 & $2.22(1.11-4.46)$ & $1.70(0.74-3.89)$ \\
\hline \multirow[t]{3}{*}{ Chromosomal } & None & 587676 & 607 & 1.00 & 1.00 \\
\hline & Any & 11903 & 17 & $1.38(0.85-2.24)$ & $1.06(0.61-1.85)$ \\
\hline & Codeine & 6524 & 11 & $1.63(0.90-2.97)$ & $1.28(0.65-2.53)$ \\
\hline \multirow[t]{4}{*}{ Oral clefts } & None & 587676 & 610 & 1.00 & 1.00 \\
\hline & Any & 11903 & 16 & $1.30(0.79-2.13)$ & $0.73(0.37-1.41)$ \\
\hline & Codeine & 6524 & 7 & $1.03(0.49-2.18)$ & $0.36(0.10-1.29)$ \\
\hline & Tramadol & 781 & $5-6$ & $6.20(2.56-14.99)$ & $1.46(0.23-9.50)$ \\
\hline \multirow[t]{2}{*}{ Respiratory } & None & 587676 & 373 & 1.00 & 1.00 \\
\hline & Any & 11903 & 8 & $1.06(0.53-2.13)$ & $0.64(0.26-1.60)$ \\
\hline \multirow[t]{3}{*}{ Eye } & None & 587676 & 278 & 1.00 & 1.00 \\
\hline & Any & 11903 & 9 & $1.60(0.82-3.11)$ & $1.40(0.69-2.82)$ \\
\hline & Codeine & 6524 & $5-6$ & $1.62(0.67-3.93)$ & $1.68(0.70-4.06)$ \\
\hline \multirow[t]{2}{*}{ Ear, face and neck } & None & 587676 & 283 & 1.00 & 1.00 \\
\hline & Any & 11903 & 7 & $1.22(0.58-2.59)$ & $0.71(0.26-1.93)$ \\
\hline
\end{tabular}

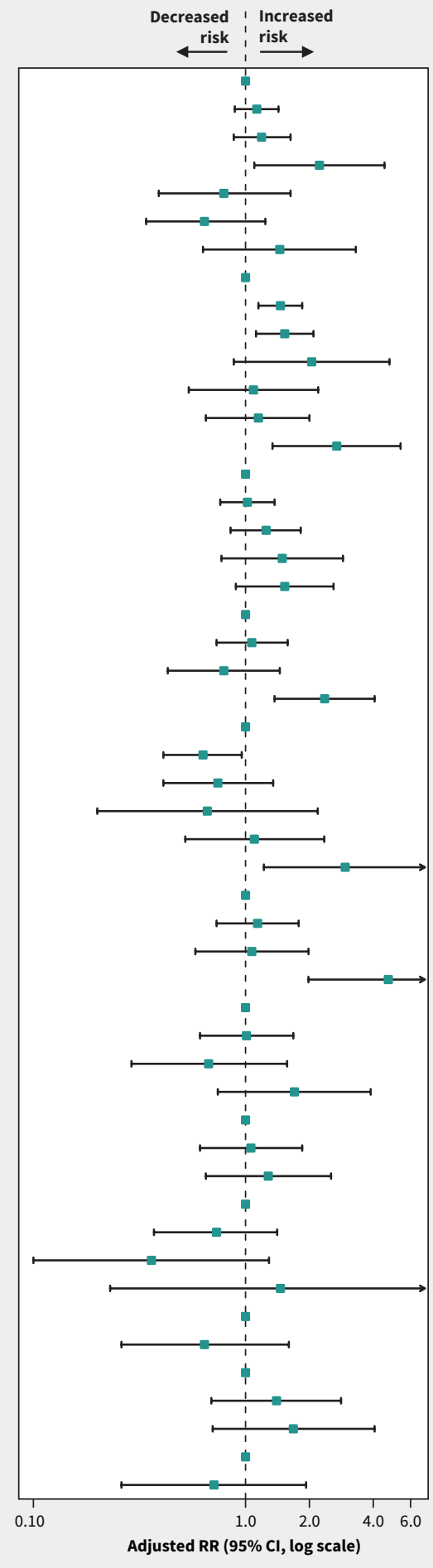

Figure 3: Forest plot of the risk of congenital anomalies in pregnancies exposed to opioid analgesics in the first trimester compared with those not exposed during the pregnancy period, classified by organ system. We adjusted estimates using inverse probability of treatment weighting with highdimensional propensity scores. *We present data on specific opioid analgesic agents where at least 5 infants with the anomaly were exposed. $\dagger$ Given ICES privacy restrictions, we report anomalies with 6 or fewer cases as 5-6. Note: $\mathrm{Cl}=$ confidence interval, $\mathrm{RR}=$ risk ratio. 


\begin{tabular}{|c|c|c|c|c|c|}
\hline \multirow[b]{2}{*}{ Major congenital anomalies } & \multirow[b]{2}{*}{ Exposure* } & \multirow{2}{*}{$\begin{array}{l}\text { No. of } \\
\text { infants }\end{array}$} & \multirow{2}{*}{$\begin{array}{l}\text { No. of infants } \\
\text { with anomalyt }\end{array}$} & \multicolumn{2}{|c|}{$\mathbf{R R}(95 \% \mathrm{Cl})$} \\
\hline & & & & Unadjusted & Adjusted \\
\hline \multirow[t]{7}{*}{ Any major congenital anomalies } & None & 587676 & 9264 & 1.00 & 1.00 \\
\hline & Any & 11903 & 247 & $1.32(1.16-1.50)$ & $1.04(0.91-1.20)$ \\
\hline & Codeine & 6524 & 121 & $1.18(0.98-1.41)$ & $1.00(0.82-1.22)$ \\
\hline & Morphine & 670 & 20 & $1.92(1.23-3.00)$ & $2.05(1.34-3.13)$ \\
\hline & Hydromorphone & 1824 & 33 & $1.15(0.81-1.62)$ & $0.85(0.56-1.27)$ \\
\hline & Oxycodone & 2885 & 66 & $1.46(1.14-1.87)$ & $0.97(0.71-1.32)$ \\
\hline & Tramadol & 781 & 30 & $2.49(1.73-3.59)$ & $1.94(1.28-2.92)$ \\
\hline \multicolumn{6}{|c|}{ Specific major anomalies (ICD-10-CA) } \\
\hline \multirow[t]{5}{*}{ Atrial septal defect (Q211) } & None & 587676 & 1961 & 1.00 & 1.00 \\
\hline & Any & 11903 & 53 & $1.34(1.02-1.76)$ & $1.22(0.91-1.62)$ \\
\hline & Codeine & 6524 & 28 & $1.29(0.89-1.87)$ & $1.15(0.78-1.72)$ \\
\hline & Oxycodone & 2885 & 13 & $1.35(0.78-2.34)$ & $0.79(0.38-1.66)$ \\
\hline & Tramadol & 781 & 9 & $3.48(1.80-6.73)$ & $2.28(0.99-5.25)$ \\
\hline \multirow[t]{4}{*}{ Ventricular septal defect (Q210) } & None & 587676 & 849 & 1.00 & 1.00 \\
\hline & Any & 11903 & 23 & $1.34(0.88-2.03)$ & $1.18(0.75-1.85)$ \\
\hline & Codeine & 6524 & 14 & $1.49(0.88-2.52)$ & $1.59(0.95-2.67)$ \\
\hline & Oxycodone & 2885 & $5-6$ & $1.20(0.50-2.89)$ & $0.61(0.17-2.21)$ \\
\hline \multirow[t]{2}{*}{ Patent ductus arteriosus (Q250) } & None & 587676 & 748 & 1.00 & 1.00 \\
\hline & Any & 11903 & 21 & $1.39(0.90-2.14)$ & $1.05(0.63-1.75)$ \\
\hline \multirow[t]{3}{*}{ Stenosis of pulmonary artery (Q256) } & None & 587676 & 311 & 1.00 & 1.00 \\
\hline & Any & 11903 & 13 & $2.06(1.19-3.60)$ & $1.81(0.99-3.31)$ \\
\hline & Codeine & 6524 & 7 & $2.03(0.96-4.29)$ & $2.16(1.03-4.51)$ \\
\hline \multirow[t]{2}{*}{ Atrioventricular septal defect (Q212) } & None & 587676 & 209 & 1.00 & 1.00 \\
\hline & Any & 11903 & $5-6$ & $1.42(0.63-3.19)$ & $0.92(0.33-2.55)$ \\
\hline \multirow[t]{2}{*}{ Tetralogy of Fallot (Q213) } & None & 587676 & 226 & 1.00 & 1.00 \\
\hline & Any & 11903 & 8 & $1.75(0.86-3.54)$ & $0.85(0.30-2.36)$ \\
\hline \multirow[t]{3}{*}{ Cleft lip (Q36) } & None & 587676 & 311 & 1.00 & 1.00 \\
\hline & Any & 11903 & 12 & $1.91(1.07-3.39)$ & $1.25(0.61-2.57)$ \\
\hline & Codeine & 6524 & $5-6$ & $1.45(0.60-3.51)$ & $0.64(0.17-2.46)$ \\
\hline \multirow{2}{*}{$\begin{array}{l}\text { Cleft palate, } \\
\text { unspecified (Q359) }\end{array}$} & None & 587676 & 205 & 1.00 & 1.00 \\
\hline & Any & 11903 & $5-6$ & $1.20(0.50-2.92)$ & $0.88(0.31-2.54)$ \\
\hline \multirow[t]{2}{*}{ Cleft palate with cleft lip (Q37) } & None & 587676 & 223 & 1.00 & 1.00 \\
\hline & Any & 11903 & 7 & $1.55(0.73-3.29)$ & $0.82(0.30-2.26)$ \\
\hline Congenital hypertrophic & None & 587676 & 728 & 1.00 & 1.00 \\
\hline \multirow[t]{4}{*}{ pyloric stenosis (Q400) } & Any & 11903 & 32 & $2.17(1.52-3.10)$ & $1.93(1.32-2.82)$ \\
\hline & Codeine & 6524 & 21 & $2.60(1.69-4.02)$ & $2.17(1.34-3.51)$ \\
\hline & Morphine & 670 & $5-6$ & $6.06(2.51-14.66)$ & $4.03(1.35-12.03$ \\
\hline & Oxycodone & 2885 & $5-6$ & $1.40(0.58-3.38)$ & $1.61(0.68-3.79)$ \\
\hline Hypospadias, & None & 587676 & 394 & 1.00 & 1.00 \\
\hline \multirow[t]{2}{*}{ balanic (Q540) } & Any & 11903 & 10 & $1.25(0.67-2.35)$ & $0.65(0.27-1.58)$ \\
\hline & Codeine & 6524 & $5-6$ & $1.14(0.47-2.76)$ & $0.75(0.25-2.25)$ \\
\hline Hypospadias, & None & 587676 & 211 & 1.00 & 1.00 \\
\hline unspecified (Q549) & Any & 11903 & $5-6$ & $1.17(0.48-2.84)$ & $0.76(0.25-2.33)$ \\
\hline \multirow[t]{3}{*}{ Congenital hydronephrosis (Q620) } & None & 587676 & 505 & 1.00 & 1.00 \\
\hline & Any & 11903 & 9 & $0.88(0.46-1.70)$ & $0.42(0.20-0.91)$ \\
\hline & Codeine & 6524 & $5-6$ & $0.89(0.37-2.15)$ & $0.45(0.13-1.59)$ \\
\hline \multirow[t]{2}{*}{ Craniosynostosis (Q750) } & None & 587676 & 299 & 1.00 & 1.00 \\
\hline & Any & 11903 & 7 & $1.16(0.55-2.45)$ & $1.22(0.58-2.56)$ \\
\hline Down syndrome, & None & 587676 & 349 & 1.00 & 1.00 \\
\hline unspecified (Q909) & Any & 11903 & 10 & $1.42(0.75-2.65)$ & $1.27(0.65-2.50)$ \\
\hline & Codeine & 6524 & $5-6$ & $1.55(0.69-3.47)$ & $1.11(0.43-2.92)$ \\
\hline
\end{tabular}

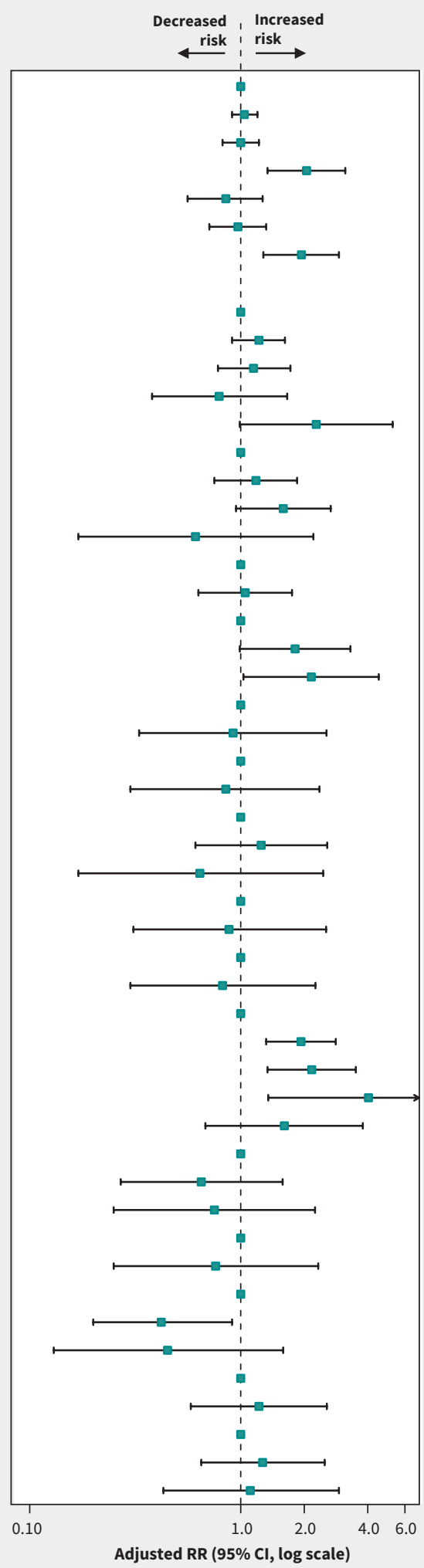

Figure 4: Forest plot of the risk of specific major congenital anomalies in pregnancies exposed to opioid analgesics in the first trimester compared with those not exposed during the pregnancy period, by specific agent. We adjusted estimates using inverse probability of treatment weighting with highdimensional propensity scores. *We present data on specific opioid analgesic agents where at least 5 infants with the anomaly were exposed. $\dagger$ Given ICES privacy restrictions, we report anomalies with 6 or fewer cases as 5-6. Note: $\mathrm{Cl}=$ confidence interval, ICD-10-CA $=$ diagnostic code from the Canadian version of the International Classification of Diseases, 10 th Revision, $\mathrm{RR}=$ risk ratio. 


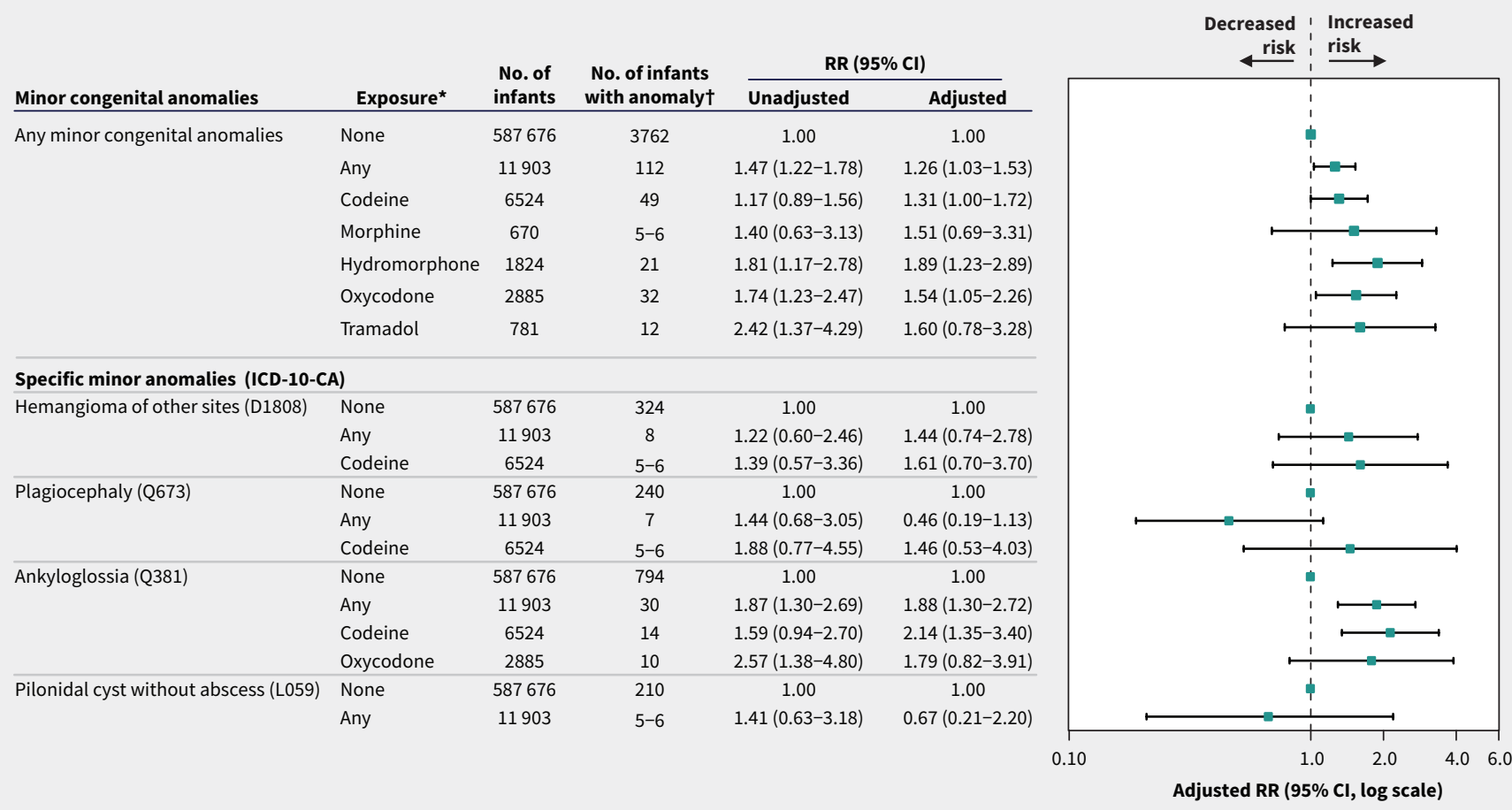

Figure 5: Forest plot of the risk of specific minor congenital anomalies in pregnancies exposed to opioid analgesics in the first trimester compared with those not exposed during the pregnancy period, by specific agent. We adjusted estimates using inverse probability of treatment weighting with highdimensional propensity scores. *We present data on specific opioid analgesic agents where at least 5 infants with the anomaly were exposed. $\dagger$ Given ICES privacy restrictions, we report anomalies with 6 or fewer cases as 5-6. Note: $\mathrm{Cl}=$ confidence interval, ICD-10-CA $=$ diagnostic code from the Canadian version of the International Classification of Diseases, 10 th Revision, $\mathrm{RR}=$ risk ratio.

were thus used as a proxy for other prenatal medications), we used psychotropic medication prevalence from a pregnant Medicaid population, ${ }^{46}$ which was higher than what would be expected in our cohort. Most associations persisted, supporting the small increased risk observed by others. ${ }^{10,14}$ Previous studies of administrative health data did not observe confounding from selective serotonin reuptake inhibitors or benzodiazepines in a high-risk cohort of pregnant people. ${ }^{31}$

Our methods improve upon some limitations of previous studies. Rather than using maternal recall, we used data from a central database that captures all prescriptions of controlled substances to capture prescriptions of opioid analgesics during pregnancy; $7,8,15-18,20,22-24,26,28,29,52$ only a small proportion of NMS records $(<3 \%)$ were not linked. Our contemporary populationbased study adds robust data to population-based studies from Sweden ${ }^{22}$ and Norway. ${ }^{24}$ Studies of Medicaid and private insurance beneficiaries often exclude many parent-infant pairs to ensure complete pregnancy coverage data; ${ }^{10,14}$ this is not a concern with our data set. Further, we systematically identified anomalies using the classification system from the Metropolitan Atlanta Congenital Defects Program.

\section{Limitations}

We identified cases using diagnostic codes for billing, which may not be entirely accurate; the diagnosis and documentation of minor anomalies and those with subtle medical significance could be vulnerable to exposure-dependent recording bias. A small number of exposed infants with certain anomalies reduced precision, which could have led to spurious associations and prevented evaluation of some previously reported associations. Deliveries before 20 weeks' gestation were not recorded; therefore, early losses and terminations (potentially owing to anomalies) were not captured. Although we had extensive prescription data, we did not have data on over-the-counter pain medications that may have been used by pregnant people. Lastly, we determined exposure by the prescription fill date without further confirmation of use; this could have attenuated our associations. Future research investigating specific anomalies and agents is warranted, given the smaller number of exposed infants.

\section{Conclusion}

Although the absolute risk of anomalies was low, our study adds to those suggesting a small increased risk of congenital anomalies with exposure to opioid analgesics. Both the potential for harm or distress to the pregnant person as a consequence of forgoing treatment and the subsequent risk to the infant must be considered for effective treatment. These findings further quantify harms associated with prenatal exposure to opioid analgesics to inform treatment choices for pain in pregnancy. 
Table 2 (part 1 of 2): Results of sensitivity analyses

\section{Analysis \\ Exposure \\ No. of infants \\ No. of anomalies}

Unadjusted RR

$(95 \% \mathrm{CI})$

Adjusted

$\operatorname{RR}(95 \% \mathrm{Cl})$

Restricting to pregnant people without opioid analgesic exposure in the previous year ${ }^{\star}$

Any congenital anomaly

\begin{tabular}{lcc} 
None & 528568 & 10483 \\
$\begin{array}{l}\text { Any opioid } \\
\text { analgesic }\end{array}$ & 6241 & 172 \\
Codeine & 3818 & 89 \\
Morphine & 398 & 18 \\
Hydromorphone & 976 & 28 \\
\hline Oxycodone & 1045 & 33 \\
\hline Tramadol & 293 & 11
\end{tabular}

Redefining exposure to include the first trimester or 4 weeks before conception

\begin{tabular}{|c|c|c|c|c|c|}
\hline \multirow[t]{7}{*}{ Any congenital anomaly } & None & 582560 & 11793 & 1.00 & 1.00 \\
\hline & Any & 17019 & 467 & $1.37(1.24-1.50)$ & $1.14(1.03-1.25)$ \\
\hline & Codeine & 9471 & 240 & $1.26(1.11-1.43)$ & $1.13(0.99-1.29)$ \\
\hline & Morphine & 799 & 27 & $1.69(1.15-2.49)$ & $1.84(1.29-2.64)$ \\
\hline & Hydromorphone & 2688 & 77 & $1.43(1.14-1.79)$ & $1.22(0.95-1.56)$ \\
\hline & Oxycodone & 4049 & 116 & $1.43(1.19-1.72)$ & $1.31(1.07-1.60)$ \\
\hline & Tramadol & 1227 & 49 & $2.01(1.51-2.68)$ & $1.66(1.20-2.29)$ \\
\hline \multicolumn{6}{|l|}{ By organ system $†$} \\
\hline Cardiovascular & Any & $3090(0.5)$ & $123(0.7)$ & $1.37(1.14-1.64)$ & $1.11(0.91-1.35)$ \\
\hline Gastrointestinal & Any & $2367(0.4)$ & $107(0.6)$ & $1.55(1.28-1.88)$ & $1.45(1.18-1.78)$ \\
\hline Musculoskeletal & Any & $1972(0.3)$ & $79(0.5)$ & $1.37(1.10-1.72)$ & $0.89(0.70-1.14)$ \\
\hline Genital & Any & $1228(0.2)$ & $47(0.3)$ & $1.31(0.98-1.76)$ & $1.12(0.81-1.55)$ \\
\hline Urinary & Any & $1333(0.2)$ & $50(0.3)$ & $1.28(0.97-1.70)$ & $0.68(0.50-0.92)$ \\
\hline Neoplasms and tumours & Any & $878(0.2)$ & $30(0.2)$ & $1.17(0.81-1.68)$ & $0.93(0.61-1.41)$ \\
\hline Central nervous system & Any & $726(0.1)$ & $30(0.2)$ & $1.42(0.98-2.04)$ & $1.02(0.67-1.56)$ \\
\hline Chromosomal & Any & $598(0.1)$ & $26(0.2)$ & $1.49(1.01-2.21)$ & $1.10(0.69-1.76)$ \\
\hline Oral clefts & Any & $604(0.1)$ & $22(0.1)$ & $1.25(0.82-1.91)$ & $0.92(0.55-1.53)$ \\
\hline Respiratory & Any & $370(0.1)$ & $11(0.1)$ & $1.02(0.56-1.85)$ & $0.59(0.26-1.31)$ \\
\hline Eye & Any & $272(<0.1)$ & $15(0.1)$ & $1.89(1.12-3.18)$ & $1.55(0.86-2.78)$ \\
\hline Ear, face and neck & Any & $274(<0.1)$ & $16(0.1)$ & $2.00(1.21-3.31)$ & $1.40(0.76-2.59)$ \\
\hline Major congenital anomalies & Any & $9160(1.6)$ & $351(2.1)$ & $1.32(1.18-1.47)$ & $1.05(0.94-1.18)$ \\
\hline Minor congenital anomalies & Any & $3712(0.6)$ & $162(1.0)$ & $1.50(1.28-1.76)$ & $1.13(0.95-1.33)$ \\
\hline \multicolumn{6}{|l|}{ Specific major anomalies (ICD-10-CA) $\dagger$} \\
\hline Atrial septal defect (Q211) & Any & $1934(0.3)$ & $80(0.5)$ & $1.42(1.13-1.77)$ & $1.18(0.92-1.51)$ \\
\hline Ventricular septal defect (Q210) & Any & $839(0.1)$ & $33(0.2)$ & $1.35(0.95-1.91)$ & $1.19(0.81-1.74)$ \\
\hline Patent ductus arteriosus (Q250) & Any & $738(0.1)$ & $31(0.2)$ & $1.44(1.00-2.06)$ & $0.97(0.62-1.52)$ \\
\hline Stenosis of pulmonary artery (Q256) & Any & $307(0.1)$ & $17(0.1)$ & $1.90(1.16-3.09)$ & $1.47(0.84-2.59)$ \\
\hline Atrioventricular septal defect (Q212) & Any & $206(<0.1)$ & $9(0.1)$ & $1.50(0.77-2.92)$ & $0.63(0.22-1.79)$ \\
\hline Tetralogy of fallot (Q213) & Any & $224(<0.1)$ & $10(0.1)$ & $1.53(0.81-2.88)$ & $0.67(0.26-1.77)$ \\
\hline Cleft lip (Q36) & Any & $309(0.1)$ & $14(0.1)$ & $1.55(0.91-2.65)$ & $1.16(0.61-2.18)$ \\
\hline Cleft palate, unspecified (Q359) & Any & $205(<0.1)$ & $5-6 \ddagger(<0.1)$ & $0.83(0.34-2.03)$ & $0.60(0.20-1.75)$ \\
\hline Cleft palate with cleft lip (Q37) & Any & $220(<0.1)$ & $10(0.1)$ & $1.56(0.83-2.93)$ & $1.09(0.50-2.36)$ \\
\hline $\begin{array}{l}\text { Congenital hypertrophic pyloric } \\
\text { stenosis (Q400) }\end{array}$ & Any & $44(0.3)$ & $716(0.1)$ & $2.11(1.55-2.86)$ & $1.99(1.45-2.73)$ \\
\hline Hypospadias, balanic (Q540) & Any & $14(0.1)$ & $390(0.1)$ & $1.23(0.72-2.09)$ & $0.82(0.42-1.60)$ \\
\hline
\end{tabular}


Table 2 (part 2 of 2): Results of sensitivity analyses

\begin{tabular}{|c|c|c|c|c|c|}
\hline Analysis & Exposure & No. of infants & No. of anomalies & $\begin{array}{l}\text { Unadjusted RR } \\
\qquad(95 \% \mathrm{CI})\end{array}$ & $\begin{array}{l}\text { Adjusted } \\
\text { RR }(95 \% \mathrm{CI})\end{array}$ \\
\hline Hypospadias, unspecified (Q549) & Any & $7(<0.1)$ & $209(<0.1)$ & $1.15(0.54-2.44)$ & $0.68(0.25-1.85)$ \\
\hline Congenital hydronephrosis (Q620) & Any & $15(0.1)$ & $499(0.1)$ & $1.03(0.62-1.72)$ & $0.34(0.19-0.61)$ \\
\hline Craniosynostosis (Q750) & Any & $9(0.1)$ & $297(0.1)$ & $1.04(0.53-2.01)$ & $1.26(0.68-2.34)$ \\
\hline Down syndrome, unspecified (Q909) & Any & $14(0.1)$ & $345(0.1)$ & $1.39(0.81-2.37)$ & $1.21(0.67-2.18)$ \\
\hline \multicolumn{6}{|l|}{ Specific minor anomalies (ICD-10-CA) } \\
\hline Hemangioma of other sites (D1808) & Any & $9(0.1)$ & $323(0.1)$ & $0.95(0.49-1.85)$ & $1.11(0.59-2.08)$ \\
\hline Plagiocephaly (Q673) & Any & $10(0.1)$ & $237(<0.1)$ & $1.44(0.77-2.72)$ & $0.22(0.10-0.49)$ \\
\hline Ankyloglossia (Q381) & Any & $37(0.2)$ & $787(0.1)$ & $1.61(1.16-2.24)$ & $1.57(1.12-2.22)$ \\
\hline Pilonidal cyst without abscess (L059) & Any & $8(<0.1)$ & $208(<0.1)$ & $1.32(0.65-2.67)$ & $0.71(0.27-1.88)$ \\
\hline \multicolumn{6}{|l|}{ Preterm births excluded (ICD-10-CA) } \\
\hline \multirow[t]{2}{*}{ Atrial septal defect (Q211) } & None & 551987 & 1277 & 1.00 & 1.00 \\
\hline & Any & 10711 & 30 & $1.21(0.84-1.74)$ & $0.96(0.65-1.44)$ \\
\hline \multirow[t]{2}{*}{ Ankyloglossia (Q381) } & None & 551987 & 697 & 1.00 & 1.00 \\
\hline & Any & 10711 & 21 & $1.55(1.01-2.40)$ & $1.89(1.27-2.81)$ \\
\hline \multirow[t]{2}{*}{ Hypertrophic pyloric stenosis (Q400) } & None & 551987 & 662 & 1.00 & 1.00 \\
\hline & Any & 10711 & 28 & $2.18(1.49-3.19)$ & $1.93(1.32-2.82)$ \\
\hline \multicolumn{6}{|l|}{ Sex-specific models } \\
\hline \multirow{2}{*}{$\begin{array}{l}\text { Female infants, hypertrophic pyloric } \\
\text { stenosis }\end{array}$} & None & 281577 & 131 & 1.00 & 1.00 \\
\hline & Any & 5687 & $5-6 \dagger$ & $2.27(1.00-5.15)$ & $3.97(2.11-7.50)$ \\
\hline \multirow{2}{*}{$\begin{array}{l}\text { Male infants, hypertrophic pyloric } \\
\text { stenosis }\end{array}$} & None & 297413 & 588 & 1.00 & 1.00 \\
\hline & Any & 5981 & 25 & $2.12(1.42-3.16)$ & $1.49(0.92-2.41)$ \\
\hline
\end{tabular}

Note: $\mathrm{Cl}=$ confidence interval, ICD-10-CA = diagnostic code from the Canadian version of the International Classification of Diseases, 10 th Revision, RR = risk ratio.

*109 859 participants had > 1 pregnancy. Of these, 106290 were unexposed in all pregnancies, 589 were exposed in all pregnancies; 2980 had at least 1 exposed and 1 unexposed pregnancy and were included in the above result.

†Number (\%) of infants reflects number of exposed infants among all 599579 infants included in sensitivity analysis. Number (\%) of anomalies reflects number of exposed infants with anomaly among 17019 exposed infants with any congenital anomaly in sensitivity analysis. Reference group for RRs is infants with no opioid analgesic exposure.

‡Because of ICES privacy restrictions, we report anomalies with 6 or fewer cases as 5-6.

\section{References}

1. Brogly SB, Turner S, Lajkosz K, et al. Infants born to opioid-dependent women in Ontario, 2002-2014. J Obstet Gynaecol Can 2017;39:157-65.

2. Camden A, Ray JG, To T, et al. Prevalence of prenatal opioid exposure in Ontario, Canada, 2014-2019. JAMA Netw Open 2021;4:e2037388-e.

3. Desai RJ, Hernandez-Diaz S, Bateman BT, et al. Increase in prescription opioid use during pregnancy among Medicaid-enrolled women. Obstet Gynecol 2014; 123:997-1002.

4. Knoppert D. The worldwide opioid epidemic: implications for treatment and research in pregnancy and the newborn. Paediatr Drugs 2011;13:277-9.

5. Zipursky J, Juurlink DN. Opioid use in pregnancy: an emerging health crisis. Obstet Med 2021;14:211-9.

6. Brogly SB, Velez MP, Werler MM, et al. Prenatal opioid analgesics and the risk of adverse birth outcomes. Epidemiology 2021;32:448-56.

7. Broussard CS, Rasmussen SA, Reefhuis J, et al. Maternal treatment with opioid analgesics and risk for birth defects. Am J Obstet Gynecol 2011;204:314 e1-11.

8. Marsh CA, Cragan JD, Alverson CJ, et al. Case-control analysis of maternal prenatal analgesic use and cardiovascular malformations: Baltimore-Washington Infant Study. Am J Obstet Gynecol 2014;211:404.e1-e9.

9. Pritham UA, McKay L. Safe management of chronic pain in pregnancy in an era of opioid misuse and abuse. J Obstet Gynecol Neonatal Nurs 2014;43:554-67.

10. Bateman BT, Hernandez-Diaz S, Straub L, et al. Association of first trimester prescription opioid use with congenital malformations in the offspring: population based cohort study. BMJ 2021;372:n102.

11. Chan F, Koren G. Is periconceptional opioid use safe? Can Fam Physician 2015; 61:431-3.
12. Lind JN, Interrante JD, Ailes EC, et al. Maternal use of opioids during preg nancy and congenital malformations: a systematic review. Pediatrics 2017;139:e20164131.

13. Yazdy MM, Desai RJ, Brogly SB. Prescription opioids in pregnancy and birth outcomes: a review of the literature. J Pediatr Genet 2015;4:56-70.

14. Wen X, Belviso N, Murray E, et al. Association of gestational opioid exposure and risk of major and minor congenital malformations. JAMA Netw Open 2021; 4:e215708-e.

15. Bracken MB, Holford TR. Exposure to prescribed drugs in pregnancy and asso ciation with congenital malformations. Obstet Gynecol 1981;58:336-44.

16. Källén B, Reis M. Use of tramadol in early pregnancy and congenital malformation risk. Reprod Toxicol 2015;58:246-51.

17. Rothman KJ, Fyler DC, Goldblatt A, et al. Exogenous hormones and other drug exposures of children with congenital heart disease. Am J Epidemiol 1979; 109:433-9.

18. Zierler S, Rothman KJ. Congenital heart disease in relation to maternal use of Bendectin and other drugs in early pregnancy. N Engl J Med 1985;313:347-52.

19. Fishman B, Daniel S, Koren G, et al. Pregnancy outcome following opioid exposure: a cohort study. PLoS One 2019;14:e0219061.

20. Yazdy MM, Mitchell AA, Tinker SC, et al. Periconceptional use of opioids and the risk of neural tube defects. Obstet Gynecol 2013;122:838-44.

21. Saxen I. Associations between oral clefts and drugs taken during pregnancy. Int J Epidemiol 1975;4:37-44.

22. Källén $B, B o r g ~ N$, Reis $M$. The use of central nervous system active drugs during pregnancy. Pharmaceuticals (Basel) 2013;6:1221-86.

23. Werler MM, Yazdy MM, Kasser JR, et al. Medication use in pregnancy in relation to the risk of isolated clubfoot in offspring. Am J Epidemiol 2014;180:86-93. 
24. Nezvalova-Henriksen $\mathrm{K}$, Spigset O, Nordeng H. Effects of codeine on pregnancy outcome: results from a large population-based cohort study. Eur J Clin Pharmacol 2011;67:1253-61.

25. Jick H, Holmes LB, Hunter JR, et al. First-trimester drug use and congenital disorders. JAMA 1981;246:343-6.

26. Shaw GM, Todoroff K, Velie EM, et al. Maternal illness, including fever, and medication use as risk factors for neural tube defects. Teratology 1998;57:1-7.

27. Bracken MB. Drug use in pregnancy and congenital heart disease in offspring. N Engl J Med 1986;314:1120.

28. Shaw GM, Malcoe LH, Swan SH, et al. Congenital cardiac anomalies relative to selected maternal exposures and conditions during early pregnancy. Eur J Epidemiol 1992;8:757-60.

29. Interrante JD, Ailes EC, Lind JN, et al. Risk comparison for prenatal use of analgesics and selected birth defects, National Birth Defects Prevention Study 1997-2011. Ann Epidemiol 2017;27:645-653.e2.

30. Patel K, Shapiro DE, Brogly SB, et al. Prenatal protease inhibitor use and risk of peterm birth among HIV-infected women initiating antiretroviral drugs during pregnancy. J Infect Dis 2010;201:1035-44.

31. Brogly SB, Hernández-Diaz S, Regan E, et al. Neonatal outcomes in a Medicaid population with opioid dependence. Am J Epidemiol 2018;187:1153-61.

32. Haight SC, Ko JY, Tong VT, et al. Opioid use disorder documented at delivery hospitalization - United States, 1999-2014. MMWR Morb Mortal Wkly Rep 2018;67:845-9.

33. Margulis AV, Setoguchi S, Mittleman MA, et al. Algorithms to estimate the beginning of pregnancy in administrative databases. Pharmacoepidemiol Drug Saf 2013;22:16-24.

34. Friend S, Richman S, Bloomgren G, et al. Evaluation of pregnancy outcomes from the Tysabri(R) (natalizumab) pregnancy exposure registry: a global, observational, follow-up study. BMC Neurol 2016;16:150.

35. Sibiude J, Le Chenadec J, Bonnet D, et al. In utero exposure to zidovudine and heart anomalies in the ANRS French perinatal cohort and the nested PRIMEVA randomized trial. Clin Infect Dis 2015;61:270-80.

36. Williams PL, Crain MJ, Yildirim C, et al. Congenital anomalies and in utero antiretroviral exposure in human immunodeficiency virus-exposed uninfected infants. JAMA Pediatr 2015;169:48-55.

37. Correa A, Cragan J, Kucik J. Reporting birth defects surveillance data 19682003. Birth Defects Res A Clin Mol Teratol 2007;79:65-186.
38. Metropolitan Atlanta Congenital Defects Program. Atlanta: Centers for Disease Control and Prevention. Available: https://www.cdc.gov/ncbddd/birthdefects/ MACDP.html (accessed 2020 May. 1).

39. Schneeweiss S, Rassen JA, Glynn RJ, et al. High-dimensional propensity score adjustment in studies of treatment effects using health care claims data. Epidemiology 2009;20:512-22.

40. Brown HK, Ray JG, Wilton AS, et al. Association between serotonergic antidepressant use during pregnancy and autism spectrum disorder in children. JAMA 2017;317:1544-52.

41. Vigod SN, Gomes T, Wilton AS, et al. Antipsychotic drug use in pregnancy: high dimensional, propensity matched, population based cohort study. BMJ 2015; 350:h2298.

42. Crump RK, Hotz JK, Imbens GW, et al. Dealing with limited overlap in estimation of average treatment effects. Biometrika 2009;96:187-99.

43. Austin PC, Stuart EA. Moving towards best practice when using inverse probability of treatment weighting (IPTW) using the propensity score to estimate causal treatment effects in observational studies. Stat Med 2015; 34:3661-79.

44. VanderWeele TJ, Ding P. Sensitivity analysis in observational research: introducing the E-value. Ann Intern Med 2017;167:268-74

45. Lash TL, Fox MP, MacLehose RF, et al. Good practices for quantitative bias analysis. Int J Epidemiol 2014;43:1969-85.

46. Huybrechts KF, Palmsten K, Avorn J, et al. Antidepressant use in pregnancy and the risk of cardiac defects. N Engl J Med 2014;370:2397-407.

47. Perinatal health indicators for Canada 2017: a report from the Canadian Perinatal Surveillance System. Ottawa: Public Health Agency of Canada; 2017.

48. Werler MM. Congenital malformations and consequential epidemiology. Curr Epidemiol Rep 2015;2:8-12.

49. Garfield K, Sergent SR. Pyloric stenosis. StatPearls 2021 July 22 [last updated].

50. Krogh C, Fischer TK, Skotte L, et al. Familial aggregation and heritability of pyloric stenosis. JAMA 2010;303:2393-9.

51. To T, Wajja A, Wales PW, et al. Population demographic indicators associated with incidence of pyloric stenosis. Arch Pediatr Adolesc Med 2005;159:520-5

52. van Gelder MM, van Rooij IA, de Walle HE, et al. Maternal recall of prescription medication use during pregnancy using a paper-based questionnaire: a validation study in the Netherlands. Drug Saf 2013;36:43-54.
Competing interests: Susan Brogly reports honoraria from the National Institutes of Health and a grant from the Canadian Institutes of Health Research, outside the submitted work. No other competing interests were declared.

This article has been peer reviewed.

Affiliations: Department of Public Health Sciences (Bowie), Queen's University, Kingston, Ont.; Department of Epidemiology (Werler), Boston University School of Public Health, Boston, Mass.; ICES (Velez, Li, Guttmann, Brogly), Kingston, Ont.; Department of Obstetrics and Gynaecology (Velez), Queen's University, Kingston, Ont.; Dalla Lana School of Public Health (Camden), University of Toronto, Toronto, Ont.; The Hospital for Sick Children (Camden, Guttmann), Toronto, Ont.; Department of Surgery (Brogly), Queen's University, Kingston, Ont.

Contributors: Martha Werler, Maria Velez, Astrid Guttmann and Susan Brogly contributed to the conception and design of the work. Wenbin Li, Astrid Guttmann and Susan Brogly contributed to the acquisition of the data. Alexa Bowie and Wenbin Li contributed to the analysis of the work. All authors contributed to the interpretation of data for the work. Alexa Bowie and Susan Brogly drafted the manuscript, and all authors revised critically for important intellectual content. All authors gave final approval of the version to be published and agreed to be accountable for all aspects of the work.

Content licence: This is an Open Access article distributed in accordance with the terms of the Creative Commons Attribution (CC BY-NC-ND 4.0) licence, which permits use, distribution and reproduction in any medium, provided that the original publication is properly cited, the use is noncommercial (i.e., research or educational use), and no modifications or adaptations are made. See: https://creativecommons. org/licenses/by-nc-nd/4.0/

Funding: This study received funding from the Eunice Kennedy Shriver National Institutes of Child Health and Human Development under grant number 1R03HD099617-01.

Data sharing: The full list of diagnosis codes and anomalies is available by request to the authors. The data set from this study is held securely in coded form at ICES. Although data sharing agreements prohibit ICES from making the data set publicly available, access may be granted to those who meet prespecified criteria for confidential access, available at www.ices.on.ca/DAS. The full data set creation plan and underlying analytic code are available from the authors upon request, understanding that the programs may rely upon coding templates or macros that are unique to ICES.

Disclaimer: This study was supported by ICES, which is funded by an annual grant from the Ontario Ministry of Health (MOH). Parts of this material are based on data and information compiled and provided by $\mathrm{MOH}$, the Canadian Institute of Health Information $(\mathrm{ClHI})$ and the Registrar General through Service Ontario. Parts of this material are based on data or information compiled and provided by $\mathrm{ClHI}$. However, the analyses, conclusions, opinions and statements expressed in the material are those of the authors, and not necessarily those of $\mathrm{CIHI}$. The analyses, conclusions, opinions and statements expressed herein are solely those of the authors and do not reflect those of the funding or data sources; no endorsement is intended nor should be inferred. The authors thank IQVIA Solutions Canada Inc. for use of their Drug Information File.

Accepted: Dec. 16, 2021

Correspondence to: Susan Brogly, susan.brogly@queensu.ca 